\title{
Efficiency Evaluation of Food and Beverage Companies in Thailand: An Application of Relational Two-Stage Data Envelopment Analysis
}

\author{
Sudarin Rodmanee and Wen-Chi Huang, Member, IACSIT
}

\begin{abstract}
This study used a relational two-stage data envelopment analysis (DEA) to evaluate the efficiency of 23 food and beverage companies in Thailand in 2011. The whole production process of the companies can be divided into two sub-processes for analyzing the efficiency differences: production achievement and profit generation. The conclusion shows that the low efficiency score of the profit generation process leads to the low overall efficiency score of the companies.
\end{abstract}

Index Terms - Food and beverage, data envelopment analysis (DEA), relational two-stage DEA, efficiency evaluation.

\section{INTRODUCTION}

The food and beverage sector contributes to the Thai national income, employment, value added inducement, and foreign exchange earning [1]. Thailand food industry provides employment to approximately 600,000 people. Today, Thailand is the sole net food exporter in Asia and has the capacity to produce far more than its consumes, earning the country the sobriquet "Kitchen of the World" [2].

Thailand's food processing industry has developed rapidly throughout the past decade and is one of the most developed in South East Asia. There are over 10,000 food and beverage factories consisting of small, medium and large-scale plants. Most of the small to medium sized businesses, serve mostly the domestic market, while medium to large food processors tend to produce higher-valued products for the domestic and export markets [3].

The food processing sector is heavily export-oriented with more than $50 \%$ of production sold outside the country. Its food exports bring in about US $\$ 10$ billion annually and comprise up to $28.3 \%$ of gross domestic product (GDP) [4].

Increasing worldwide competition and proliferation of consumer requirements have been posing significant challenges for Thai food and beverage companies to remain competitive in the global market. Thai firms are already encountering competition from other countries such as Vietnam and China, who have abundant resources and lower labor costs. To maintain Thailand's position as a leader in the food processing efficiency is important.

The performance evaluation is a major concern of top

Manuscript received December 15, 2012; revised April 30, 2013.

Sudarin Rodmanee is with Department of Tropical Agriculture and International Cooperation, National Pingtung Unversity of Science and Technology, Nonthaburi, Thailand (e-mail: sudarin@ hotmail.com). management in the food and beverage industry. Since it is imperative to find a tool to help firms to understand their deficiencies and identify industry leaders to benchmark. Therefore, this paper aims to evaluate efficiency of the food and beverage companies in Thailand by using the relational two-stage data envelopment analysis (DEA).

\section{LITERATURE REVIEW}

Among direct approaches used to measure efficiency performance, non-parametric data envelopment analysis (DEA) has been widely used. Many studies split the whole operational process into sub-processes and explore which sub-process causes inefficiency.

The simplest case of a whole production process is divided into two sub-processes. The decision making units (DMUs) can have a two-stage structure. The first stage uses inputs to generate outputs which become the inputs to the second stage. The first stage outputs are therefore called intermediate measures. The second stage then uses these intermediate measures to produce outputs.

The two-stage DEA method was firstly introduced to analyze the financial efficiency of commercial bank's production process which was divided into the stage of profitability and marketability [5]. The two-stage DEA method for calculating the efficiencies of the sub-processes does not reflect any relationship between components and the whole system.

However, the relational two-stage DEA was then suggested to apply to non-life insurance companies in Taiwan [6]. Under the relational two-stage DEA method, the overall efficiency is the product of the efficiencies of the two sub-processes, and the efficiencies calculated from this approach are more meaningful than those calculated from the independent two-stage DEA approach [7].

The whole production process of the food and beverage companies can be divided into two-sub processes: production achievement and profit generation. Since the company has a typical two-stage structure of the production process, the methodology of relational two-stage DEA approach discussed in the previous section can be applied to measure the efficiencies of the whole process and the two sub-processes. Hence, the results from the efficiency evaluation could provide managerial insights that allow decision makers in the 
company to make improvements.

\section{METHODS}

\section{A. Data Collection}

Considering the effectiveness of getting the data, deliberating the size of the company and the degree of competitiveness in the international markets, this paper selects the 26 food and beverage companies in Thailand, which are listed on the Stock Exchange of Thailand in 2011. These companies are the key players in the Thai food industry.

Because the Thai food industry is very competitive, there were 25 companies that had positive net profit in year 2011 . For obtaining more meaningful results and after deleting 2 companies with missing data, we collected the cross-sectional data of 23 companies from the Stock Exchange of Thailand in 2011 [8] to perform efficiency measurement. The selected companies included CFRESH, CPF, F\&D, HTC, LST, MALEE, MINT, OISHI, PB, PM, PR, S\&P, SAUCE, SFP, SORKON, SSC, SSF, TC, TF, TIPCO, TUF, TVO, and TWFP. For anonymity reason, these companies were represented by S1 S23 without following the aforementioned order hereafter.

\section{B. Analysis Approach Using Relational Two-Stage DEA}

This paper applies the relational two-stage DEA method proposed by Kao and Hwang [6] to decompose and measure the efficiencies of the companies under investigation.

The whole production process of food and beverage companies is composed of a two sub-processes. The process 1 is production achievement and the process 2 is profit generation. The efficiency of the first stage measures the performance in producing and selling products of the company while the efficiency of the second stage measures the performance in generating profit from the domestic market and foreign market.

The whole process uses $m$ inputs $X_{i k}, i=1, \ldots, m$ to produce $s$ outputs $Y_{r k}, r=1, \ldots, s$. The whole production process is composed of two sub-processes with $q$ intermediate products $Z_{p k}, p=1, \ldots, q[6]$. The intermediate products $Z_{p k}$ are the outputs of stage 1 as well as the inputs of stage 2 .

To link the two sub-processes with the whole process, the model must describe the series relationship between the whole process and the two sub-processes. Consider DMU $k$. Denote $u_{r}{ }^{*}, v_{i}{ }^{*}$, and $w_{p}{ }^{*}$ as the multipliers that DMU $k$ has selected to calculate its overall efficiency $E_{k}$ and sub-process efficiencies $E_{k}{ }^{l}$ and $E_{k}{ }^{2}$. Based upon the CCR-model, the overall efficiency and sub-processes efficiencies can be expressed as the following

$$
\begin{aligned}
& E_{k}=\sum_{r=1}^{s} u_{r} \times Y_{r k} / \sum_{i=1}^{m} v_{i} \times X_{i k} \leq 1 \\
& E_{k}^{1}=\sum_{r=1}^{s} w_{p} \times Z_{p k} / \sum_{i=1}^{m} v_{i} \times X_{i k} \leq 1
\end{aligned}
$$

$$
E_{k}^{2}=\sum_{r=1}^{s} u_{r} \times Y_{r k} / \sum_{p=1}^{q} w_{p} \times Z_{p k} \leq 1
$$

The overall efficiency is the product of the efficiencies of stage 1 and 2: $E_{k}=E_{k}{ }^{l} \times E_{k}{ }^{2}$.

In this study, the input-oriented model was selected because it is easier for company managers to manage and control inputs.

\section{Inputs, Intermediate and Outputs}

The inputs of the whole process, which are also the inputs of the first stage, are: cost of sales and services $\left(X_{1}\right)$ and other expenses $\left(X_{2}\right)$. The outputs of the whole process, which are also the outputs of the second stage, are: net profit $\left(Y_{1}\right)$ and other comprehensive income $\left(Y_{2}\right)$. There are two intermediate products in the whole process, which are the outputs of the first stage as well as the inputs of the second stage: revenues from sales and services $\left(Z_{1}\right)$ and other income $\left(Z_{2}\right)$.

\section{RESULTS AND MANAGEMENT IMPLICATIONS}

The overall effectiveness, production efficiency, and profitability efficiency evaluated by the relational two-stage DEA are summarized in Table I.

\begin{tabular}{|c|c|c|c|c|}
\hline DMUs & $E_{k}$ & $E_{k}^{l}$ & $E_{k}$ & \\
\hline $\mathrm{S} 1$ & $0.1282(17)^{\mathrm{a}}$ & $0.9782 \quad(8)$ & 0.1310 & (18) \\
\hline $\mathrm{S} 2$ & 0.2827 & $0.9426 \quad(12)$ & 0.2999 & (9) \\
\hline $\mathrm{S} 3$ & $0.1259 \quad(18)$ & $0.9234 \quad(15)$ & 0.1363 & (17) \\
\hline $\mathrm{S} 4$ & $0.0330 \quad(23)$ & 1.0000 & 0.0330 & (23) \\
\hline S5 & $0.0718 \quad(20)$ & $0.9209 \quad(16)$ & 0.0780 & (20) \\
\hline S6 & $0.1385 \quad(16)$ & $0.8221 \quad(22)$ & 0.1685 & (14) \\
\hline S7 & $0.4474 \quad(5)$ & 1.0000 & 0.4474 & (6) \\
\hline S8 & 0.4061 & $0.8542 \quad(19)$ & 0.4755 & (5) \\
\hline S9 & 0.8891 & $0.9267 \quad(14)$ & 0.9593 & (3) \\
\hline S10 & $0.3605 \quad(8)$ & $0.8807 \quad(18)$ & 0.4094 & (7) \\
\hline S11 & $0.9014 \quad$ (2) & $0.9014 \quad(17)$ & 1.0000 & (1) \\
\hline $\mathrm{S} 12$ & $0.2284 \quad(10)$ & $0.9419 \quad(13)$ & 0.2425 & (10) \\
\hline S13 & 0.5791 & 1.0000 & 0.5791 & (4) \\
\hline S14 & $0.1401 \quad(15)$ & 0.9729 (9) & 0.1440 & (15) \\
\hline S15 & $0.1006 \quad(19)$ & $0.8266 \quad(21)$ & 0.1217 & (19) \\
\hline S16 & $0.0552 \quad(21)$ & $0.7830 \quad(23)$ & 0.0705 & (21) \\
\hline S17 & 0.2250 & $0.9696 \quad(10)$ & 0.2321 & (11) \\
\hline S18 & $0.0500 \quad(22)$ & 0.9888 & 0.0506 & (22) \\
\hline S19 & $0.3708 \quad(7)$ & 1.0000 & 0.3708 & (8) \\
\hline $\mathrm{S} 20$ & $0.1587 \quad(13)$ & $0.8487 \quad(20)$ & 0.1870 & (13) \\
\hline $\mathrm{S} 21$ & $0.1961 \quad(12)$ & $0.9436 \quad(11)$ & 0.2078 & (12) \\
\hline $\mathrm{S} 22$ & $0.1421 \quad$ (14) & 1.0000 & 0.1421 & (16) \\
\hline $\mathrm{S} 23$ & 1.0000 & 1.0000 & 1.0000 & (1) \\
\hline Average Scores & 0.3138 & 0.9315 & 0.3255 & \\
\hline $\begin{array}{l}\text { No. of Efficiency } \\
\qquad(\text { Score }=1)\end{array}$ & 1 & 6 & \multicolumn{2}{|l|}{2} \\
\hline
\end{tabular}

TABLE I: EFFICIENCY MEASURES OF THE 23 FOOD AND BEVERAGE COMPANIES IN THAILAND 
For the whole process, only S23 is efficient. Moreover, S9 and S11 have an efficiency score of greater than 0.8. These three companies are considered as companies having good performance. On the other hand, there are fifteen companies, whose efficiency scores are less than average value 0.3138 , are considered as companies having unsatisfactory performance. The results imply that there is still room for improvement.

The productivity and profitability can be a mirror of an efficiency difference among companies. For stage 1, the sub-process of production achievement, there are six companies, which perform efficiently. For stage 2, the sub-process of profit generation, there are only two companies, namely S11 and S23, which have a perfect efficiency. The results reveal that very few food and beverage companies have efficiently generated profit from their products.

From the performance decomposition results (Table I.), most companies have a higher $E_{k}{ }^{1}$ than $E_{k}{ }^{2}$. Only S9 and S11 have a smaller $E_{k}{ }^{1}$ than $E_{k}^{2}$. The efficiencies of two sub-processes also show that the production achievement process performs relatively better than the profit generation process. Their averages are 0.9315 and 0.3255 , respectively. This indicates that the low efficiency score of the whole process is mainly due to the low efficiency score of the second stage, i.e., the profit generation sub-process.

According to the empirical results summarized above, a management decision matrix is developed to serve as a basis for an evaluation of good and poor performance among the food and beverage companies at different stages. The management decision matrix is created by comparing the efficiency scores of two sub-processes of each company with its average efficiency scores of stage 1 and 2.

The two sub-processes are divided into two quadrants: high and low using its average efficiency scores as a criterion. Crossing the strategic variables results gives four quadrants that represent the company position and what strategy is needed to improve overall efficiency. Production achievement is on the $\mathrm{x}$ axis and profit generation is on the $\mathrm{y}$ axis. The companies are discriminated by their efficiency scores and categorized into four groups, as shown in Fig. 1.

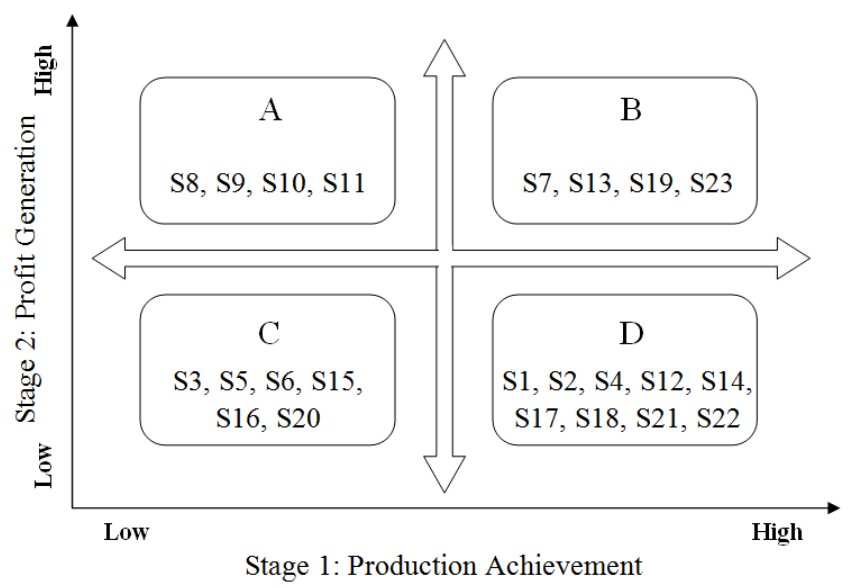

Fig. 1. Management decision matrix for managerial efficiency.

The four companies in Group A (Low-High) are more efficient than the average score in the profit generation efficiency score, but lower than the average in the production achievement efficiency score. This type has good profitability but poor productivity. All they need to do is to improve specially their productivity strategies. Group B (High-High) contains four companies. Their production efficiency and profitability efficiency are higher than the average efficiency scores of production achievement and profitability generation. They have advantages both in production efficiency and profitability efficiency. There are six companies in Group C (Low-Low), showing lower scores than the average efficiency values both in production achievement and profit generation. These companies should simultaneously strengthen the efficiency both in production efficiency and profitability efficiency. Finally, the nine companies in Group D (High-Low) are higher in the production efficiency but lower than the average profitability efficiency value. This type should attempt to maintain their production advantages whilst also try to enhance their profit generating strategies.

\section{CONCLUSIONS}

This paper uses the relational two-stage DEA to measure 23 food and beverage companies in Thailand. It provides detailed results on the efficiencies of the whole process and the two sub-processes, namely production achievement and profit generation. The results show that most of the companies have low overall efficiency scores. There is only one company perform efficiently both in stage 1 and stage 2 . The efficiency of the first stage is much higher than the second stage, implying that the low efficiency score of the profit generation leads to the low overall efficiency score of the whole process. Therefore, managers should simultaneously consider productivity and profitability improvement.

Under intense international competition nowadays, the efficiency measurement of food and beverage industry is necessary to ensure the survival and development of company. The relational two-stage DEA approach is a powerful tool for measuring efficiency. It provides useful information to assist managerial decision making in order to improve the performance of food and beverage companies in Thailand.

\section{ACKNOWLEDGMENT}

Sudarin Rodmanee wishes to thank Taiwan Scholarship for supporting scholarships in pursing the study and sincerely thank the Ministry of Agriculture and Cooperatives, Thailand for giving an opportunity to continue education in graduate study.

\section{REFERENCES}

[1] S. Lekuthai, "The importance of the food industry to the Thai economy: an input-output perspective," ASEAN Economic Bulletin, vol. 24, pp. 238-253, 2007.

[2] E. V. Murray, "Thailand-the kitchen of the world, origin and growth of the Thai food industry and lessons for India," CAB Calling, pp. 16-26, April-June 2007.

[3] U.S. Department of Agriculture, Foreign Agricultural Service, "Thailand exporter guide 2010, GAIN report number: TH1005," Office of Agricultural Affairs U.S. Embassy, Bangkok, Thailand, 2011.

[4] Thailand Board of Investment, "Food industry," Bangkok, Thailand, 2008. 
[5] L. M. Seiford and J. Zhu, "Profitability and marketability of the top 55 US commercial banks," Management Science, vol. 45, pp. 1270-1288, 1999.

[6] C. Kao and S. N. Hwang, "Efficiency decomposition in two-stage data envelopment analysis: an application to non-life insurance companies in Taiwan," European Journal of Operational Research, vol. 185, pp. 418-429, 2008.

[7] S. T. Liu and R. T. Wang, "Efficiency measures of PCB manufacturing firms using relational two-stage data envelopment analysis," Expert System with Applications, vol. 36, pp. 4935-4939, 2009.

[8] The Stock Exchange of Thailand. (2012). Companies/securities info. [Online].

Available: http://www.set.or.th/set/todaynews.do?language=en\&country=US .

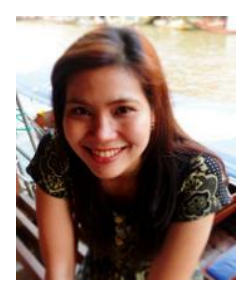

Sudarin Rodmanee was born in Bangkok, Thailand on November 1, 1980. Finished Bachelor of Biotechnology, 2002, Kasetsart University, Bangkok, Thailand. Master of Food Science, National Pingtung University of Science and Technology, Pingtung, Taiwan. In present, Ph.D. Candidate on Agribusiness at Department of Tropical Agriculture and International Cooperation, National Pingtung University of Science and Technology, Pingtung, Taiwan. She is a Researcher at Research and Planning Bureau, Agricultura Land Reform Office, Ministry of Agriculture and Cooperatives, Thailand. Her research interest is agribusiness, community enterprise, public health and food safety. 